\title{
UMA ABORDAGEM PARA TROCA DE MENSAGENS USANDO ARQUÉTIPOS
}

\author{
An Approach for Message Exchange Using Archetypes \\ João Luís Cardoso de Moraes'; Wanderley Lopes de Souza²; Luciana Tricai Cavalini³; \\ Luís Ferreira Pires"; Antônio Francisco do Prado²
}

\begin{abstract}
Resumo A aplicação das TICs na área de cuidado de saúde, conhecida como e-Health, pode facilitar o acesso aos serviços de cuidado de saúde e só será aceitável se permitir a troca de informação eficiente e segura entre os cuidadores de saúde e pacientes. O objetivo deste trabalho é apresentar uma abordagem para troca de mensagens em cenários realísticos.
\end{abstract}

Palavras-chave: : Arquétipos, openEHR, Interoperabilidade Semântica

\begin{abstract}
The application of ICT in healthcare sector activities, known as e-Health, can simplify the access to healthcare services and will only be acceptable for realistic scenarios if it supports efficient and secure information exchange amongst the caregivers and their patients. The aim of this paper is to present an approach for message exchange to realistic scenarios.
\end{abstract}

Keywords: Archetypes, openEHR, Semantic Interoperability

\section{Introdução}

O uso de Tecnologias da Informação e Comunicação (TIC) para apoiar práticas de saúde (e-Health) ${ }^{1}$ auxilia no acesso aos serviços de cuidado de saúde, com potenciais benefícios para os usuários desses serviços. A troca de informações entre sistemas de Registro Eletrônico de Saúde (RES) heterogêneos em ambientes de cuidado de saúde requer padrões de comunicação que permitam a interoperabilidade entre esses sistemas. OpenEHR² é uma Fundação dedicada à pesquisa de RESs interoperáveis e definiu uma arquitetura aberta baseada em modelagem de dois níveis que separa as informações de conhecimento. A principal motivação deste trabalho foi explorar se os arquétipos que definem os conceitos clínicos podem ser usados para a troca de mensagens entre RESs heterogêneos, possibilitando atingir um nível adequado de interoperabilidade semântica.

\section{Relato da Experiência}

A arquitetura openEHR foi desenvolvida com base no paradigma de modelagem de dois níveis, conforme mostrado na Figura 1. No primeiro nível, um Modelo de Referência (RM) foi definido como um conjunto predefinido de classes que modelam a estrutura de um RES; e no segundo nível, definiram-se conceitos específicos, restringindo as classes do RM em termos de arquétipos, expressos em Linguagem

1. Pós-graduando da Universidade Federal de São Carlos; 2. Professor da Universidade Federal de São Carlos; 3. Professor da Universidade Federal Fluminense; 4. Professor at University of Twente - PhD 
de Definição de Arquétipo (ADL) ${ }^{3}$. Um arquétipo é um modelo formal de um conceito de um domínio e espera-se que seja facilmente compreensível por um especialista do domínio.



Figura 1. Modelo Dual openEHR

Clinical Knowledge Manager (CKM) ${ }^{4}$ é um repositório de arquétipos, proposto pela fundação openEHR, contendo um conjunto de arquétipos que representam conceitos clínicos e podem ser reutilizados em várias aplicações do domínio de saúde. Neste projeto, foram reutilizados alguns arquétipos providos pelo CKM, como Device, Device Details e Clinical Synopsis, mas também foram desenvolvidos novos arquétipos para representar conceitos clínicos do domínio de cardiologia, como Pacemaker Implantation,
Vascular Cardiac Surgery, Coronary Cardiac Surgery, Angioplasty Cardiac e Pacemaker Evaluation.

A abordagem proposta foi desenvolvida com base no modelo openEHR e como uma aplicação de software integrando RESTful Webservices ${ }^{5}$. A Figura 2 mostra uma visão geral do esquema do Gerador de Mensagens contendo um Esquema do Modelo de Referência (RM-XMLSchema) para representar as restrições do RM openEHR e um Esquema do Modelo de Arquétipos (AM-XMLSchema) para representar os arquétipos contendo conceitos clínicos. O Gerador de Mensagens implementa as classes do RM e usa eXtensible Markup Language (XML) para geração de extratos de RES contendo informações clínicas de pacientes (vide Figura 2).

\section{Discussão}

O Centro de Cirurgia Cardíaca de Marília (CCM) realiza implante de marcapasso em pacientes na região de Marília/SP e dispõe de um RES para documentar os cuidados de saúde prestados aos pacientes. O Gerador de Mensagem possibilita a reunião de informações de saúde do paciente que irá implantar um marcapasso. Neste cenário realístico, Dr. Call está perto de implan-

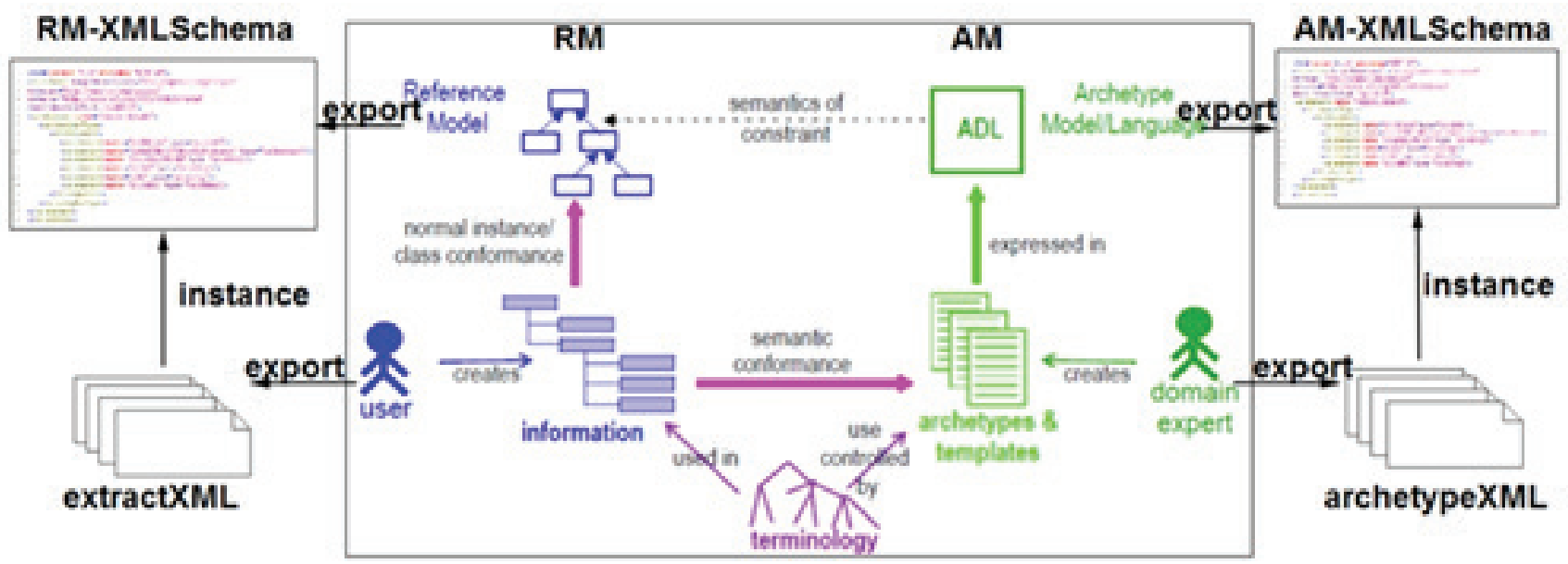

Figura 2. Esquema do Gerador de Mensagens 
tar um marcapasso no paciente Sr. Martins. A Figura $3^{1}$ mostra Dr. Call requisitando informações do paciente para planejar a cirurgia. O Gerador de Mensagem processa a requisição e as informações clínicas são recuperadas (2a-d) de vários RES distribuídos e o Gerador de Mensagens serializa Extratos de RES contendo informações requisitadas e enviadas ${ }^{3}$ então para o Dr. Call. O Gerador de Mensagens foi testado e avaliado por profissionais de saúde, mostrando a usabilidade e aceitação da proposta por parte dos usuários finais (vide Figura 3).

\section{Comentários Finais}

Este trabalho apresentou uma abordagem para troca de mensagens que usa o padrão openEHR. O Gerador de Mensagens foi projetado com base no modelo openEHR e fornece uma infraestrutura para permitir a interoperabilidade semântica no domínio de saúde. O estudo de caso apresentando foi conduzido em um ambiente de cuidado de saúde distribuído realístico envolvendo cinco provedores de cuidado de saúde na região de Marília/SP. Os serviços RESTful utilizados na abordagem padronizam os mecanismos para descrição, descoberta e acesso aos recursos providos pelo Gerador de Mensagens, permitindo que RESs heterogêneos possam interoperar por meio da troca de mensagens XML. O uso do Gerador de Mensagens em um cenário realístico mostrou a usabilidade e aceitação da abordagem proposta por parte dos cuidadores de saúde uma vez que permite aos profissionais de saúde reunir informações relacionadas aos seus pacientes em um ambiente de cuidado de saúde distribuído.

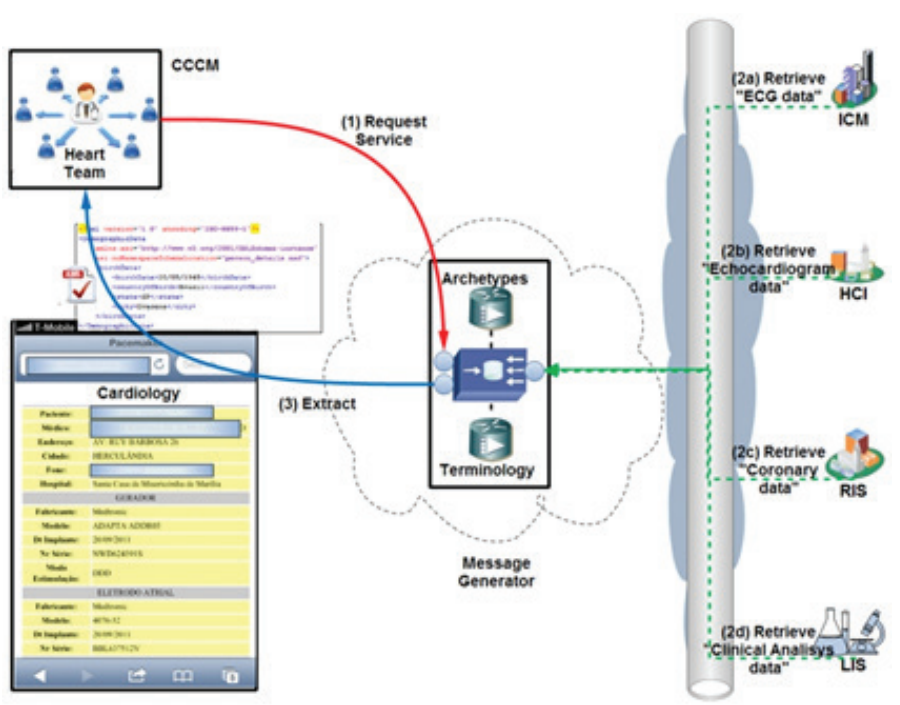

Figura 3. Caso de Uso do Gerador de Mensagens

\section{Referências}

1. DeLuca JM, Enmark R. E-health: the changing model of healthcare. Frontiers of health services management. 2000;17(1):3-15. PubMed PMID: MEDLINE:11184427.

2. Beale T. Archetypes and the EHR. Advanced Health Telematics and Telemedicine: the Magdeburg Expert Summit Textbook. 2003;96:238-44. PubMed PMID: WOS:000189484900032.

3. Beale T, Heard S. Archetype Definition Language. openEHR Foundation, 2007.

4. openEHR-CKM. openEHR Foundation - Clinical Knowledge Manager 2013 [cited 2014 07/02/2014]. Available from: http://openehr.org/ckm/.

5. Richardson L, Ruby S. RESTful Web Services. First Edition edition ed: O'Reilly Media; 2007. $454 p$ 